Forum 2017 · 32:449

https://doi.org/10.1007/s12312-017-0349-0

o) Springer Medizin Verlag GmbH 2017

\title{
Modelle für eine bessere Medizin
}

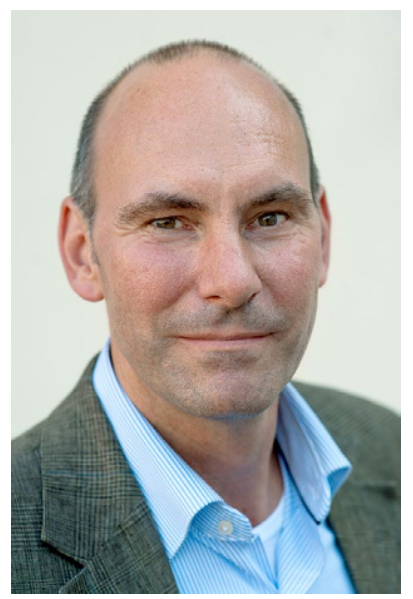

Die akademische Medizin definiert Fortschritt vorwiegend über den Erkenntnisgewinn zu pathophysiologisch relevanten Krankheitsmerkmalen und deren therapeutischer Beeinflussung. Die täglich am Patienten tätig werdenden Ärzte und Gesundheitsberufe sehen meistens einen mindestens gleich großen Bedarf in Verbesserungen der Versorgungsstrukturen. Wie können wir eine Gesundheitsversorgung entwickeln, die mit größter Wahrscheinlichkeit nutzbringend und wirksam ist, aber von gesetzlichen Krankenversicherungen bisher nicht bezahlt wird?

Der Innovationsfonds fördert seit 2016 neue Versorgungsformen, die über die bisherige Regelversorgung hinausgehen, sowie Versorgungsforschungsprojekte, die auf neue Erkenntnisse zur bestehenden Versorgung in der Gesetzlichen Krankenversicherung (GKV) ausgerichtet sind. Auch 10 onkologische Projekte werden derzeit gefördert. Die Beiträge von Professor Hecken, Vorsitzender des G-BA und Dr. Pollandt, Anwaltskanzlei Sträter, Bonn, erörtern die gesetzlichen Hintergründe, Zielsetzung, Struktur und Arbeitsweise des Innovationsfonds. Der Innovationsfonds wurde für die Jahre 2016 bis 2019 mit jährlich 300 Mio. EUR ausgestattet. Das Geld stammt aus Liquiditätsreserven des Gesundheitsfonds sowie von den GKV. Der Innovationsfonds ist damit eine Investition der Beitragszah- ler in die Weiterentwicklung der Versorgungsstrukturen der GKV. Die Beteiligung der Krankenkassen an Struktur- und Prozessentwicklung im Gesundheitssystem ist neu und bietet Chancen. Wie diese genutzt werden und ob es zur Implementierung neuer Leistungen in die Regelversorgung kommt, muss abgewartet werden.

Eine der größten Herausforderungen für die Zukunft des Gesundheitssystems ist die nutzbringende Verwendung digital gespeicherter Informationen für die Prävention, die Behandlung von Erkrankungen und die Forschung. Digital Health fasst nach Definition der WHO die Bereiche eHealth (electronic health) und $m$ Health (mobile health) zusammen. Mit eHealth wird die sichere und kosteneffektive Nutzung von Informations- und Kommunikationstechnologien für die Gesundheit bezeichnet. Der Begriff mHealth bezieht sich auf eHealth-Anwendungen, die Gesundheitsdienste und Informationen über mobile Technologien wie Smartphones und Tablets zur Verfügung stellen; dazu zählen u.a. Gesundheits- und Medizin-Apps. Mit der Digitalisierung sind große Erwartungen an eine Individualisierung von Präventions- und Versorgungskonzepten und die Verbesserung von Qualität und Effizienz in der Leistungserbringung verknüpft. Computergestützte Prozesse und Analysen wecken die Hoffnung auf bürokratische Entlastung und auf neue Freiräume für die Arzt-Patient-Beziehung. Voraussetzung dafür sollen mit einer sicheren Telematikinfrastruktur geschaffen werden, die Daten über Sektorengrenzen hinweg sammeln und austauschen kann. Die Entwicklungen stehen am Anfang. Welche Vorteile letztlich wirklich für die Behandlung, die Patientensicherheit und den Komfort und die Effizienz der Behandelnden resultieren, wird die weitere Entwicklung zeigen. Was es für Krebspatienten bedeuten könnte, wenn ihre in elektronischen Symptomtagebüchern erfassten Daten in einer Patientenakte sicher abgelegt, von Pflegenden und Ärzten zeitnah eingesehen und zur Beratung genutzt werden, zeigen Er- gebnisse aus jüngsten Studien. Demnach sind Vorteile für Lebensqualität und sogar für das Überleben denkbar [Basch E et al. JAMA 2017]. Ein Beitrag von Dr. Kramer, Freiburg und Professor Vollmar, Jena fasst den Entwicklungsstand von Digital Health in der Onkologie zusammen.

Mit FARKOR - Vorsorge bei familiärem Risiko für das kolorektale Karzinom (Dr. Christa Maar, München) und CARE for CAYA - Ganzheitliches Präventionsprogramm für junge Patienten nach überstandener Krebserkrankung im Kindes-, Jugendund jungen Erwachsenenalter (Dr. Julia Quidde und Dr. Alexander Stein, Hamburg) stellen wir exemplarisch für andere wertvolle Projekte in diesem Forum zwei vom Innovationsfonds geförderte onkologische Versorgungsmodelle vor. Die Projekte verfolgen das Ziel, junge Menschen mit familiär bedingt erhöhtem Krebsrisiko sinnvollen Früherkennungsmaßnahmen zuzuführen (FARKOR) bzw. bei jungen Krebsüberlebenden auf die speziellen Bedürfnisse dieser Patientengruppe einzugehen und eine umfassende und individualisierte Nachsorge anzubieten (CARE for CAYA).

Wir wünschen den hier vorgestellten wie auch anderen innovativen Modellprojekten in Deutschland einen gewinn- und erkenntnisbringenden Verlauf und sehen hoffnungsvoll effizienteren und Patientenorientierten Versorgungsstrukturen in der Krebsmedizin entgegen.

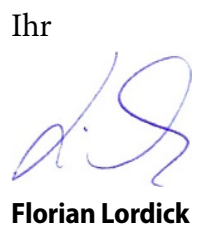

\section{Korrespondenzadresse}

\section{Universitäres Krebszentrum Leipzig}

(UCCL)

Prof. Dr. med. Florian Lordick

Liebigstraße 20

04103 Leipzig, Deutschland

Tel:0341 9712560

E-Mail: Direktion.UCCL@medizin.uni-leipzig.de 